\title{
Disrafismo Espinal Oculto: Reporte de Caso
}

\section{Occult Spinal Dysraphism: Case Report}

\author{
Janina Franco-Chávez $\mathbb{1}^{1}$, Nathalia Belén Méndez-González $\mathbb{D}^{1}$, Oscar Ucedo ${ }^{1}$.
}

${ }^{1}$ Universidad Nacional de Asunción, Facultad de Ciencias Médicas, Santa Rosa del Aguaray, Paraguay.

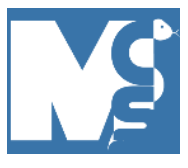

Recibido: $18 / 07 / 2021$

Revisado: $12 / 08 / 2021$

Aceptado:30/08/2021

\section{Autor correspondiente}

Janina Franco-Chávez

Universidad Nacional de Asunción,

Paraguay

luzjanina98@gmail.com

\section{Conflictos de interés}

Los autores declaran no poseer conflictos de interés.

\section{Fuente de financiación}

Los autores no recibieron apoyo financiero para la investigación, autoría y/o publicación de este artículo.

Este artículo es publicado bajo una licencia de Creative Commons Reconocimiento 4.0 Internacional.

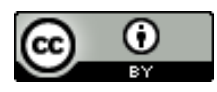

\section{RESUMEN}

El disrafismo espinal oculto incluye alteraciones en la fusión del tubo neural, en el que la lesión está cubierta por la epidermis, su incidencia estimada es de 0,5 a 5 casos por cada 1000 nacidos vivos. Los estigmas cutáneos pueden ser el único signo de la patología, los cuales pueden no ser muy evidentes. Reporte de caso de mujer de 36 años que acudió a consulta por cuadro de dolor lumbar de inicio insidioso, de 3 semanas de duración, que con el paso de los días se fue intensificando, irradia a miembros inferiores, cede parcialmente con analgésicos comunes y se acompaña de parestesias en ambos miembros. Se solicitó TAC y RMN que reveló anomalía anatómica sacrococcígea caracterizada por abertura de canal raquídeo, ausencia de cóccix, situación baja del cono medular, siringomielia e hiperintensidad a nivel del filum terminal sugestivo de lipoma. Se confirmó el diagnóstico de espina bífida oculta a nivel sacro, con lipoma del filum terminal, médula anclada con situación baja del cono medular y siringomielia asociada. Actualmente es controversial el beneficio cirugía profiláctica en pacientes asintomáticos, siendo preferible una conducta conservadora con estrecho seguimiento urológico y neurológico, entre las medidas de prevención se recomienda evitar el ejercicio físico extenuante, disminuir carga de columna lumbar y reeducación postural.

Palabras clave: Espina bífida oculta; Disrafia Espinal; Defectos del tubo neural..

\section{ABSTRACT}

Occult spinal dysraphism includes alterations in the fusion of the neural tube, in which the lesion is covered by the epidermis, its estimated incidence is 0.5 to 5 cases per 1000 live births. Cutaneous stigmata may be the only sign of pathology, which may not be very obvious. Case report of a 36 -year-old woman who came to the clinic for insidious onset lumbar pain, lasting 3 weeks, which intensified over the days, radiating to the lower limbs, partially subsided with common analgesics and accompanied by paresthesias in both limbs. CT and MRI are requested, revealing sacrococcygeal anatomical abnormality characterized by spinal canal opening, absence of coccyx, low location of the medullary cone, syringomyelia, and hyperintensity at the level of the terminal filum suggestive of lipoma. The diagnosis of occult spina bifida at the sacral level was confirmed, with a lipoma of the terminal filum, an anchored cord with a low location of the medullary cone and associated syringomyelia. Currently, the benefit of prophylactic surgery in asymptomatic patients is controversial, being preferable a conservative behavior with close urological and neurological monitoring, among the prevention measures it is recommended to avoid strenuous physical exercise, reduce lumbar spine load and postural reeducation.

Keywords: Spina bifida occulta; Spinal Dysraphism; Neural tube defects. 


\section{INTRODUCCIÓN}

El disrafismo espinal oculto o espina bífida oculta incluye alteraciones en la fusión del tubo neural, en el que la lesión está cubierta por la epidermis. Existen dos tipos de disrafismo espinal, la abierta y la oculta, aunque se desconoce la incidencia exacta de esta última se puede afirmar que es la más frecuente entre ambos tipos (1), siendo su incidencia estimada de 0,5 a 5 casos por cada 1000 nacidos vivos (2).

La piel puede tener un aspecto normal o mostrar signos sugestivos de una enfermedad subyacente; los estigmas cutáneos pueden ser el único signo de la patología, los cuales pueden no ser muy evidentes. Hasta en un $70 \%$ de los casos de espina bífida oculta aparecen alteraciones cutáneas múltiples en la línea media posterior a nivel de la lesión, asimismo, estas manifestaciones pueden aparecer aisladas 0 en cualquier región de la columna vertebral (2).

En adultos asintomáticos esta malformación puede ser de hallazgo casual que generalmente es confirmado por una Resonancia Magnética Nuclear (RMN), en estos casos y en los que cursan con síntomas leves se recomienda un tratamiento conservador y la contraindicación relativa de técnicas anestésicas en los que presentan medula anclada (3).

\section{PRESENTACIÓN DEL CASO}

Paciente de 36 años de edad, de sexo femenino, sin patología de base conocida, acude a consulta por cuadro de dolor lumbar de inicio insidioso, de 3 semanas de duración, que con el paso de los días se fue intensificando, irradia a miembros inferiores: el derecho hasta la región posterior del tobillo y el izquierdo hasta cara posterior del muslo, siendo en este último de menor intensidad, cede parcialmente con analgésicos comunes; se acompaña de parestesias en ambos miembros. Refiere que hace 3 meses presentó cuadro similar, pero de menor intensidad; además como antecedente relevante dolor lumbar, lumbociatalgia bilateral y paresia de ambos miembros, de aproximadamente 3 meses de duración, posterior a una apendicectomía. Al examen físico de la región lumbosacra: simétrico, sin abombamiento, leve cambio de coloración en región sacra, negruzco apenas visible, con desviación del pliegue interglúteo (Figura 1).

FIGURA 1. SE EVIDENCIA LIGERA DESVIACIÓN DEL PLIEGUE INTERGLÚTEO, ESTIGMA CUTÁNEO NO VISIBLE EN LA IMAGEN.

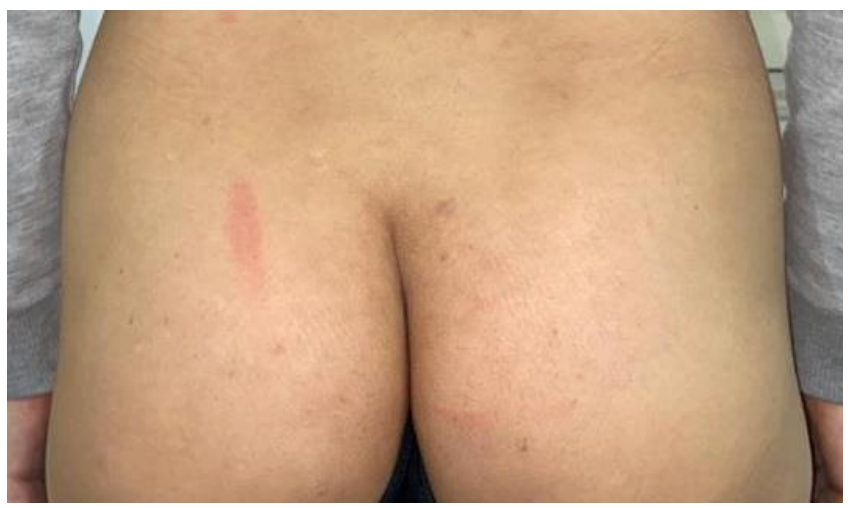

A la palpación: sacro con terminación truncada, no se palpa cóccix. De los miembros inferiores: lado derecho con sensibilidad disminuida en territorio S1, fuerza muscular 5/5, reflejos osteotendinosos presentes y conservados, Lasegue negativo; lado izquierdo sin datos de valor. Se solicita tomografía axial computarizada (TAC) simple y resonancia magnética nuclear (RMN) sin contraste de columna lumbosacra. En TAC Ilama la atención anomalía anatómica sacrococcígea caracterizada por abertura de canal raquídeo y ausencia de cóccix (Figura 2). En la RMN se observa situación baja del cono medular, siringomielia e hiperintensidad a nivel del filum terminal sugestivo de lipoma (Figura 3).

Con los datos se confirma el diagnóstico de espina bífida oculta a nivel sacro, con lipoma del filum terminal, médula anclada con situación baja del cono medular y siringomielia asociada. Debido a la ausencia de sintomatología significativa se realiza tratamiento conservador con antiinflamatorios no esteroideos por 72 horas, luego según necesidad, reeducación postural y fisioterapia; control al término de las sesiones. Se orienta a la paciente sobre signos de alarma sugestivos de complicaciones 


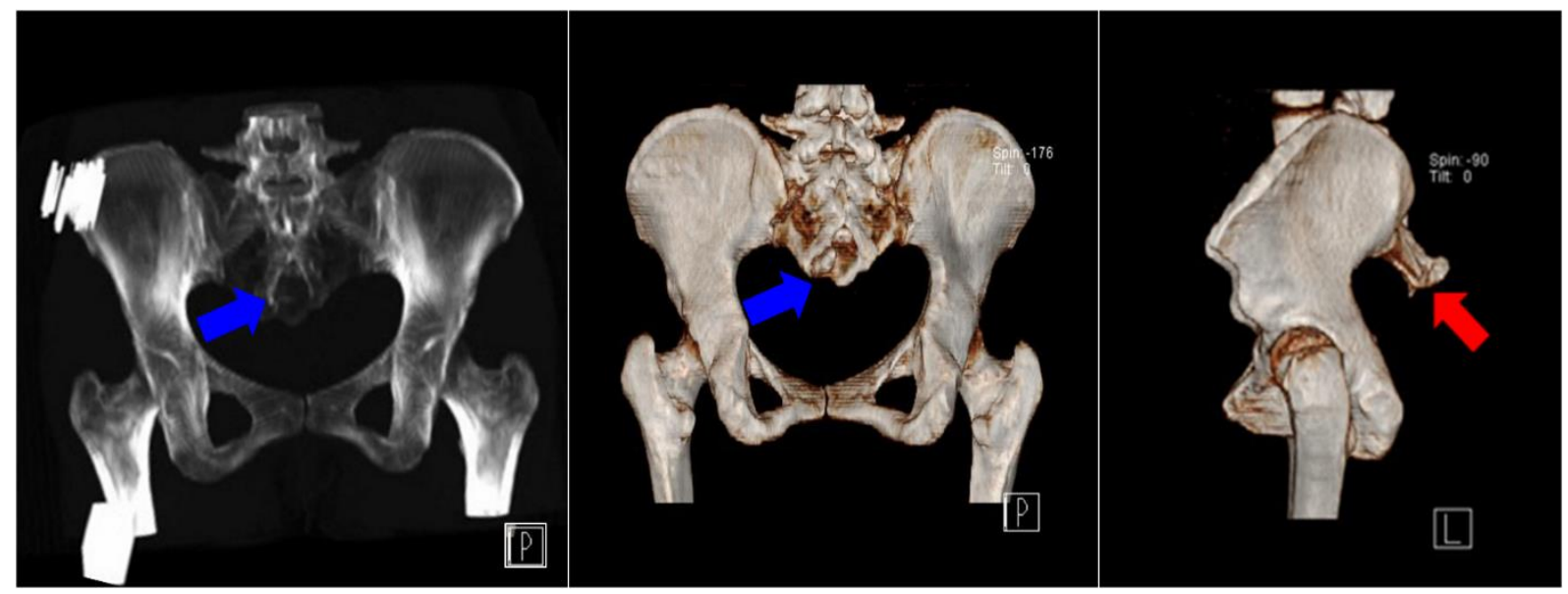

FIGURA 3: RMN DE COLUMNA LUMBOSACRA, SIN CONTRASTE, CORTES SAGITAL Y AXIAL, RESPECTIVAMENTE.

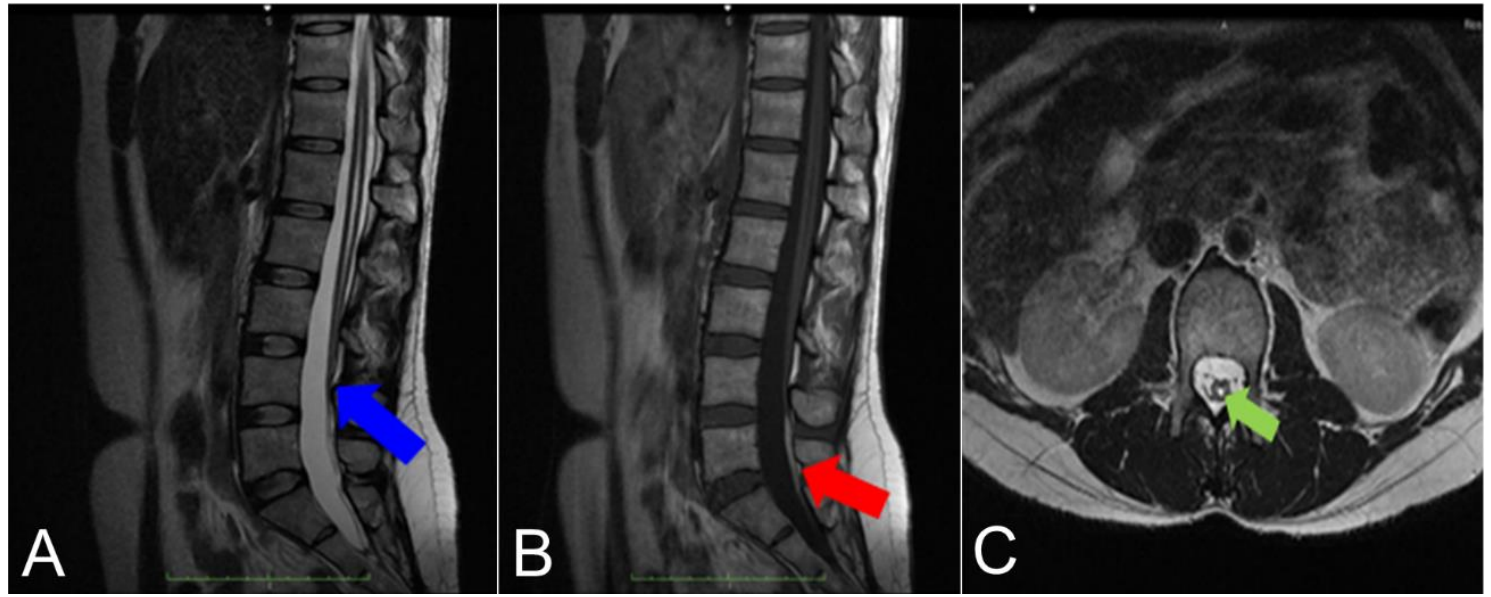

A. Se observa cono medular distal que termina a la altura de porción superior de L4, en T2 (flecha azul). B. Hiperintensidad en T1 a nivel del filum terminal, en relación con lipoma (flecha roja). C. dilatación del canal ependimario (flecha verde).

\section{DISCUSIÓN}

Entre los tipos de defectos del tubo neural la espina bífida es la de mayor prevalencia, seguida de la anencefalia y de la encefalocele; en un estudio realizado en Brasil se informó una prevalencia de Espina Bífida de 1,4 por cada 10000 nacimientos, siendo en la región de las Américas la mediana de 11,5 por 10000 nacimientos (4).

Los estigmas cutáneos se encuentran en el 2,2 al 7,2 \% de todos los recién nacidos, de los cuales solo un pequeño porcentaje presenta disrafismo oculto (5); esto debido a que la piel y el tejido nervioso comparten origen embriológico. La separación incompleta entre el ectodermo cutáneo y el tubo neural puede originar medula anclada, así como su separación prematura puede desarrollar lipoma (6). El disrafismo espinal oculto suele acompañarse de lipomas lumbosacros y generalmente se diagnostica en lactantes por alguna manifestación cutánea o por presencia de signos y síntomas en adultos jóvenes, los cuales suelen ser de carácter progresivo (5). Además, el $10 \%$ de los pacientes con antecedente de espina bífida presentan siringomielia como una de las manifestaciones neurológicas (7).

Los nervios raquídeos pueden no estar afectados en la médula anclada por espina bífida oculta y los pacientes pueden permanecer asintomáticos o presentar síntomas leves durante muchos años. Cuando se manifiestan, consisten principalmente en alteraciones sensitivas y motoras de las extremidades inferiores y debilidad de las mismas, alteraciones de los esfínteres, como vejiga neurogénica, y síntomas dolorosos en región lumbar o ciática. Las complicaciones de esta patología corresponden a la acentuación de la sintomatología ya mencionada, las cuales son potencialmente irreversibles (8).

El Gold Estándar para el diagnóstico de certeza es la RMN, la TAC ayuda a explorar las estructuras óseas y no aporta mayores datos para el diagnóstico de la patología. Anteriormente la cirugía era considerada 
tratamiento de elección incluso de manera profiláctica, pero actualmente es controversial el beneficio de la misma en pacientes asintomáticos, siendo preferible una conducta conservadora con estrecho seguimiento urológico y neurológico considerando que la cirugía se acompaña de un alto índice de complicaciones permanentes y que la mayoría de los pacientes no presentarán déficit neurológico a lo largo de su vida (2). Entre las medidas de prevención se recomienda evitar

\section{REFERENCIAS}

1. Mongay ML. Síndrome de médula anclada en el periodo neonatal: comunicación de un caso. Acta Pediátrica Esp. Disponible

en:

https://www.actapediatrica.com/index.php/secciones/not as-clinicas/1703-sindrome-de-medula-anclada-en-elperiodo-neonatal-comunicacion-de-un-caso

2. Anomalía cutánea lumbar en un lactante: ¿siempre una lesión banal? Disponible en: http://pap.es/articulo/12703/anomalia-cutanea-lumbaren-un-lactante-siempre-una-lesion-banal

3. Mesa Suárez P, Montes Durán C, Hidalgo Ramos FJ, Calvo Castilla ML. Detección de espina bífida oculta en la consulta de preanestesia. Rev Esp Anestesiol Reanim .2018;65 (3):181-2. https://dx.doi.org/10.1016/j.redar.2017.05.004

4. Describing the Prevalence of Neural Tube Defects Worldwide: A Systematic Literature Review. Disponible en: https://journals. plos.org/plosone/article?id=10.1371/jour nal.pone.0151586\#pone-0151586-g001

5. Massimi L, Feitosa Chaves TM, Legninda Sop FY, Frassanito $P$, Tamburrini G, Caldarelli M. Acute presentations of intradural lipomas: case reports and a review of the el ejercicio físico extenuante, disminuir carga de columna lumbar y reeducación postural, tal como se indicó en el caso presentado (9).

\section{CONTRIBUCIÓN DE LOS AUTORES}

Todos los autores participaron en la redacción del borrador, revisión crítica del manuscrito y aprobación final del mismo.

literature. BMC Neurol .2019;19

(1):189. https://dx.doi.org/10.1186/s12883-019-1413-4

6. Rios LTM, Oliveira RVB de, Martins M da G, Leitão OMR, Simões VMF, Nascimento JMS do. Lipoma espinhal associado a seio dérmico congênito: relato de caso. Radiol Bras .2011;44:265-7. https://dx.doi.org/10.1590/S0100$\underline{39842011000400014}$

7. Díaz Sanhueza C, Pardo Vargas RA, Bustos P. Manifestaciones neurológicas asociadas a espina bífida en adultos. Med Fam SEMERGEN .2018;44 (4):276-80. https://dx.doi.org/10.1016/j.semerg.2017.09.003

8. ICSEB. Médula anclada con espina bífida oculta - Institut Chiari de Barcelona. Inst Chiari Siringomielia Escoliosis Barc. Disponible en: https://institutchiaribcn.com/espina-bifidaoculta/

9. Li W, Xiong Z, Dong C, Song J, Zhang L, Zhou J, et al. Distribution and imaging characteristics of spina bifida occulta in young people with low back pain: a retrospective cross-sectional study. J Orthop Surg .2021;16:151. https://dx.doi.org/10.1186/s13018-021-02285-w 